\title{
Analysis of Nonstationary Transfer of 1:1 Electrolyte in Intensive Current Regimes
}

\author{
Natalia Olegovna Chubyr ${ }^{1}$, Vitaly Alexandrovich Gudza ${ }^{2}$, Makhamet Khuseevich Urtenov ${ }^{2}$, \\ Inna Vladimirovna Shkorkina ${ }^{2}$ \\ ${ }^{1}$ Kuban State Technological University, Krasnodar, Russia \\ ${ }^{2}$ Kuban State University, Krasnodar, Russia
}

\begin{abstract}
This work is aimed at mathematical simulation of nonstationary transfer of 1:1 electrolyte in depleted layer near ion-exchange membrane and determination of fundamental regularities of this process, including analysis of structure of diffusion layer and its variations in time. It is demonstrated that the diffusion layer in overlimiting regime is more complicated than in sublimiting regime, when the diffusion layer is comprised of two regions: electroneutrality region and minor boundary layer near cation-exchange membrane where the region of quasi-equilibrium space charge is generated. It is known that in overlimiting stationary regimes, the additional extended region of space charge is generated. For nonstationary problems, the interest is attracted to variations of boundaries of these regions in time, existence of asymptotics in time. This is a novelty of the nonstationary problem. This work describes classification of problems of nonstationary transfer, according to which variations of electroneutrality regions and spatial charge in time have been studied. Boundary problems are solved by finite element method.
\end{abstract}

Key words : membrane systems, diffusion layer, mathematical model, overlimiting transfer, intensive current regimes.

\section{INTRODUCTION}

Membrane systems are referred to crucially important technologies. They are used for water purification, in microfluidic devices (lab-on-a-chip), for development of new biomaterials, upon development of fuel cells, etc. Upon current passage in these systems, a diffusion layer is generated [1-9].

Levich has demonstrated 1 that when sublimiting current passes through electrode/solution, the diffusion layer is subdivided into electroneutrality region (ENR) and space charge region (SCR), whereas the latter region is quasi-equilibrium since the distribution of concentration, field intensity, etc. do not depend on current density. Rubinstein and Shtilman2 have demonstrated that in the case of overlimiting planes of current, in addition to ENR and quasi-equilibrium SCR, there appears the so-called extended SCR. Stationary problems are analyzed using decomposition method in $[3,4]$.

Nonstationary problems are studied in $[1,5,6,7]$. In these works, the main attention is paid to the analysis of startup time depending on problem variables; in $[1,4,6]$, potentiostatic regime is analyzed, when potential jump is considered as constant; and in [5], galvanostatic regime with constant current is considered. In this work, the main attention is devoted to classification of problems of nonstationary transfer, regularities of established structure of diffusion layer, and kinetic regularities of transfer of salt ions depending on the problem type.

\section{METHODS}

\subsection{Mathematical Model}

Mathematical model is comprised of a set of equations with partial derivatives of the first order, boundary and initial conditions. The equations are based on common conservation laws, thus, the classification is made with regard to boundary and initial conditions.

\subsubsection{Model equations}

It is known [8] that nonstationary 1D mathematical model of mass transfer of binary electrolyte is described as follows:

$$
\begin{gathered}
\frac{\partial C_{i}}{\partial t}=-\frac{\partial j_{i}}{\partial x} \quad i=1,2 \\
j_{i}=-\frac{F}{R T_{0}} z_{i} D_{i} C_{i} \frac{\partial \phi}{\partial x}-D_{i} \frac{\partial C_{i}}{\partial x} \quad i=1,2 \\
\frac{\partial^{2} \phi}{\partial x^{2}}=-\frac{F}{\varepsilon_{a}}\left(z_{1} C_{1}+z_{2} C_{2}\right)
\end{gathered}
$$


Natalia Olegovna Chubyr et al., International Journal of Emerging Trends in Engineering Research, 8(8), August 2020, 4337 - 4345

$$
I_{c}=F\left(z_{1} j_{1}+z_{2} j_{2}\right)
$$

where (1) are the equations of material balance, (2) are the Nernst-Planck equations for flows of sodium ions $i=1 \leftrightarrow N a^{+}$and chlorine ions $i=2 \leftrightarrow C l^{-}$, the charge numbers of cations: $z_{1}=1$, and anions: $z_{2}=-1$, (3) is the Poisson equation for potential of electric field, (4) is the equation of current passage, which means that current passing through the diffusion layer is determined by flow of ions. The constants: ${ }^{\varepsilon_{a}}$ is the dielectric permeability of solution, $F$ is the Faraday number, $R$ is the universal gas constant. The required functions: $\varphi$ is the potential, $E=-\frac{\partial \varphi}{\partial x}$ is the intensity of electric field, $C_{i}, j_{i}, D_{i}, I_{c}$ are the concentration, the flow, the diffusion coefficient of the i-th ion, the current density determined by ion flow.

\subsubsection{Boundary conditions}

From considerations of physics it follows that boundary conditions for nonstationary problem can be determined by analogue with stationary problem ${ }^{1,3}$, except for the condition for potential jump.

Let us assume that $x=0$ corresponds to the depth of solution where the condition of electroneutrality is valid, and $x=H$ is the conventional interphase boundary of solution-ion-exchange membrane (Fig. 1).

Let us assume that cation-exchange membrane (CEM) is ideally selective. In general case in the nonstationary problem, the ion concentrations deep in solution depend on time. However, for most physically interesting problems they can be considered as constant. The value of cations at the right boundary $(x=H$ ) is set equal to exchange capacity of CEM. Theoretically, the exchange capacity of membrane varies in time, however, for small time interval this variation can be neglected. It follows from ideal selectivity of CEM that anion flow through it is zero. While studying transfer of salt ions in diffusion layer, it is required to distinguish between two different regimes: potentiostatic regime, when the potential jump $\varphi(H, t)-\varphi(0, t)=\varphi_{d}$ is considered to be constant, independent on time $t$, and potentiometric regime, when the potential jump depends on time, that is $\varphi(H, t)-\varphi(0, t)=\varphi_{d}(t)$. Thus, for instance, for calculation of current-voltage characteristic (CVC), $\varphi_{d}(t)=d \cdot t$, where $d$ is the increment rate of potential jump. Since the potential function is determined with the accuracy up to arbitrary function depending on time (see Eqs.
(2) and (3)), then, without loss of generality, it is possible to assume that $\varphi(0, t) \equiv 0$, then $\varphi(H, t)=\varphi_{d}(t)$. The solution at the left boundary of diffusion layer $(x=0)$ is electrically neutral, thus, $C_{1}(0, t)-C_{2}(0, t)=0$. Hence, we have the following boundary conditions:

$$
\begin{gathered}
C_{1}(t, 0)=C_{0}(t), C_{2}(t, 0)=C_{0}(t), \varphi(t, 0)=0 \\
C_{1}(t, H)=C_{1 m}(t),\left(\frac{\partial C_{2}}{\partial x}-\frac{F}{R T_{0}} z_{2} C_{2} E\right)(t, H)=0 \\
\varphi(t, H)=\varphi_{d}(t)
\end{gathered}
$$

\subsubsection{Initial Conditions}

Formulation of initial conditions (at $t=0$ ) is significantly different depending on the research objectives, they can be classified, for instance, as follows:

1) If space charge generation is analyzed, current-voltage characteristics are calculated, or similar problems are considered, then the initial state of physical process should be sublimiting. Therefore, the initial conditions should determine sublimiting state. Such boundary conditions are, for instance, models of transfer in diffusion layer in the cross section of initial segment of desalination channel of electrodialysis apparatus [1].

2) During simulation of transfer in diffusion layer in the cross section of initial segment of desalination channel, it should be taken into account that the established transfer is already overlimiting process and, hence, the initial conditions should determine overlimiting state of the diffusion layer.

3) Mixed initial conditions, when, for instance, the initial distribution of concentration satisfies the condition of electroneutrality (which corresponds to overlimiting regime),

and $\varphi_{d}$ is so high that it corresponds to overlimiting regime. Such initial condition can occur, for instance, upon simulation of transfer using piecewise current of high intensity $[2,7,9]$.

4) Some works ${ }^{4}$ study the influence of pulse currents on transfer of salt ions in desalination channel of electrodialysis apparatus. Upon mathematical simulation of such regime in diffusion layer, the initial conditions are comprised of single localized pulses and the problem can be interpreted as distribution of pulsed currents in time.

The initial conditions in general case are written as follows:

$$
\begin{aligned}
& C_{1}(0, x)=C_{10}(x) \quad, \quad C_{2}(0, x)=C_{20}(x), \\
& \varphi(0, x)=\varphi_{0}(x)
\end{aligned},
$$


where these functions are determined according to items 1) $-3)$.

Thus, for instance, for sublimiting regime it is possible to set $C_{10}(x)=C_{0}, C_{20}(x)=C_{0}, \varphi_{0}(x)=\varphi_{0}$, where $\varphi_{0}$ is sufficiently low. Since the initial conditions satisfy the condition of electroneutrality and $\varphi_{0}$ is low, then we have sublimiting regime.

Overlimiting initial condition can be exemplified as follows:

$$
\begin{gathered}
C_{1}(0, x)=C_{10}(x)=\left\{\begin{array}{l}
\left(1-\frac{I_{i n i}}{I_{u l t}} \cdot \frac{x}{H}\right) C_{0}, \quad x \in\left[0, \frac{I_{u l t}}{I_{i n i}} H\right] \\
\frac{\sqrt{R T \varepsilon_{0} H}}{2 D_{0} F^{2} \sqrt{C_{0}}} \cdot \frac{I}{\sqrt{\frac{I}{I_{u l t}} x-H}}, \quad x \in\left[\frac{I_{u l t}}{I_{\text {ini }}} H, H\right]
\end{array}\right. \\
C_{2}(0, x)=C_{1}(0, x), \\
\phi_{0}(x)=\frac{R T}{F} \ln \left(1-\frac{I_{i n i}}{I_{u l t}} \frac{x}{H}\right), \quad x \in\left[0, \frac{I_{u l t}}{I_{i n i}} H\right) \\
\phi_{0}(x)=\phi_{d}+\frac{4}{3} \frac{\sqrt{R T C_{0}}}{\sqrt{\varepsilon_{0} H}} \frac{I_{u l t}}{I_{i n i}}\left(\frac{I_{i n i}}{I_{u l t}} x-H\right)^{\frac{3}{2}}-\frac{4}{3} \frac{\sqrt{R T C_{0}}}{\sqrt{\varepsilon_{0}}} \frac{I_{u l t} H}{I_{i n i}}\left(\frac{I_{i n i}}{I_{u l t}}-1\right)^{\frac{3}{2}}, x \in\left(\frac{I_{u l t}}{I_{i n i}} H, H\right]
\end{gathered}
$$

where $I_{\text {in }}$ is the density of current passing through the diffusion layer at initial time, herewith, $I_{i n}>I_{u l t}$.

Equations (6) - (9) were derived using the solution to stationary problem $[1,3]$.

5) Certain interest is attracted to analysis of discharge of accumulated space charge upon sudden deactivation of external source at any time $t_{\text {ini }}$. In this case, variation of potential jump can be set, for instance, initially in the form of linear function of time $t$, to certain $t_{i n i}$, and then zero.

6) Stability of transfer, calculation of impedance, etc. are analyzed using periodic regime of potential jump variation, for instance, $\varphi(t, H)=\varphi_{0}+d \cdot \sin (\omega t+\tau)$, where $\varphi_{0}, d, \omega, \tau$ are the potential jump, the amplitude, the oscillation frequency, and the phase shift

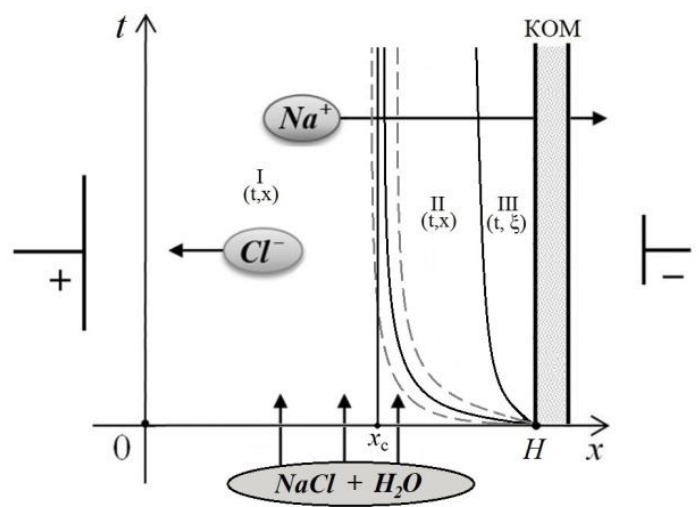

a)

\section{RESULTS}

\subsection{Results of Numerical Analysis}

Numerous calculations were performed in the work with many variables. However, typical concentrations $C_{0}=0.1$ mole $/ \mathrm{m}^{3}$, and thicknesses of diffusion layer $H=10^{-4}$ are retained.

It should be mentioned that for each regime, the calculations are performed for different number of seconds and different increment depending on the purposes.

\subsubsection{Structure of diffusion layer}

The main results of the work are illustrated in Fig. 1.

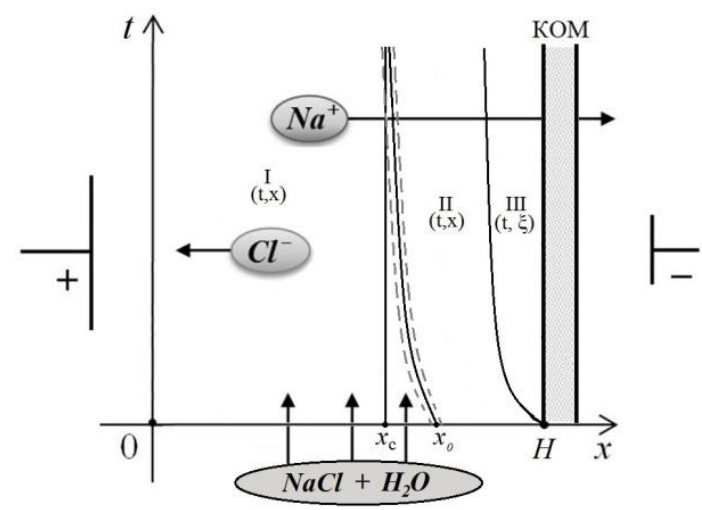

b) 


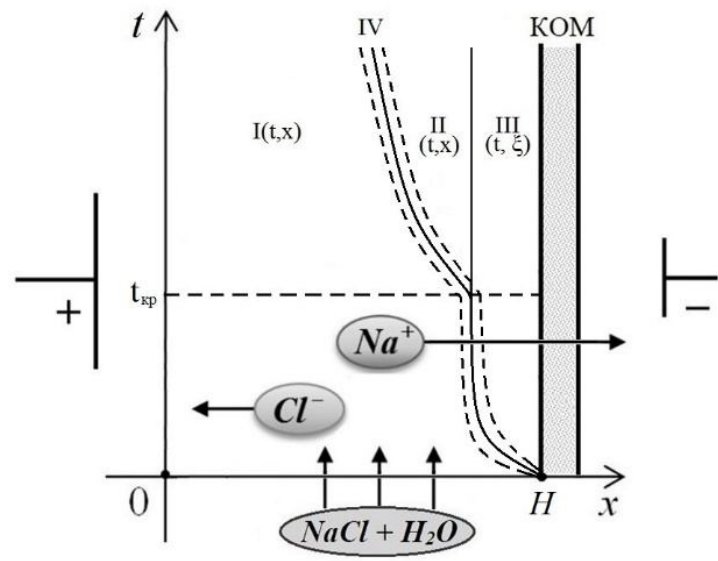

c)

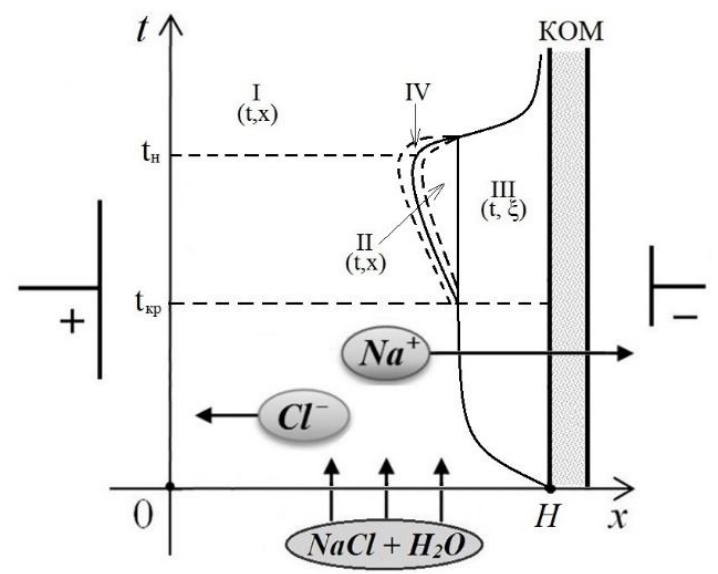

d)

Figure 1: Schematic view of diffusion layer (without scale): a) sublimiting initial state, and overlimiting continuous potential jump (overlimiting potentiostatic regime); b) overlimiting initial state and overlimiting potentiostatic regime; c) mixed initial state for calculation of CVC; d) current regime, for analysis of discharge current (see Section 5): I - ENR, II - region of extended space charge near CEM, III - region of quasi-equilibrium space charge (boundary layer near CEM)

It follows from Fig. 1 that in all cases there is the expected ENR (region 1 in the figure), where $C_{1}(t, x) \approx C_{2}(t, x)$ is, with high accuracy, the boundary layer (region III in the figure), the sizes of which do not depend on current density as shown by calculations, thus, it is referred to as quasi-equilibrium ${ }^{1,8}$ and the extended region of space charge (SCR) (region II in the figure). Near the left boundary of the extended SCR, the density of space charge has local maximum. As can be seen in Fig. 1, the left boundary of the extended SCR depends significantly on the type of boundary problem. The quasi-equilibrium boundary layer (region III) is one half of classical double electric layer ${ }^{8}$, its width approaches asymptotically at $t \rightarrow \infty$ to the Debye length.

It is aimed to substantiate these results using numerical analysis of boundary problem. The plots of

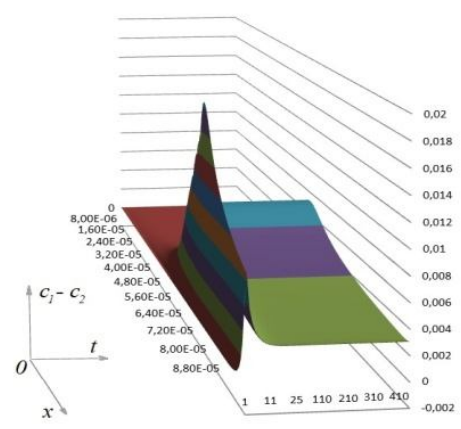

a)

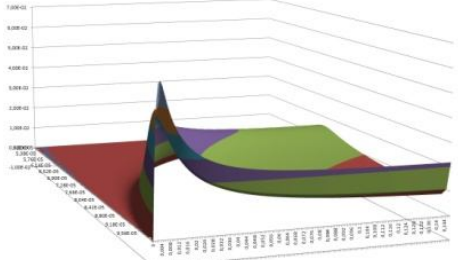

b)

$$
\frac{1}{F} \rho(t, x)=C_{1}(t, x)-C_{2}(t, x)
$$

are used (density of charge distribution normalized for convenience with respect to the Faraday number) at various angles.

Figures 2. a)-c) correspond to Fig. 1 a). For the sake of convenience, Fig. 2 a) does not depict the quasi-equilibrium SCR (III in Fig 1a) near membrane, thus, the local maximum is distinctly seen near normalized density of charge distributing. In Fig. 2 b) and especially in Fig. 2 c), the quasi-equilibrium SCR near membrane can be observed, and it is cut off from above, otherwise, the rest could not be seen. If the surface $2 \mathrm{c}$ is correctly rotated, then its respective lines of level would coincide with the lines in Fig, 1 a) when the scale is varied.

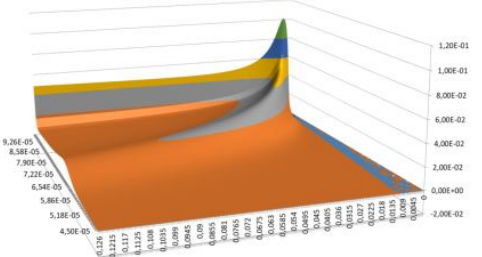

c) 


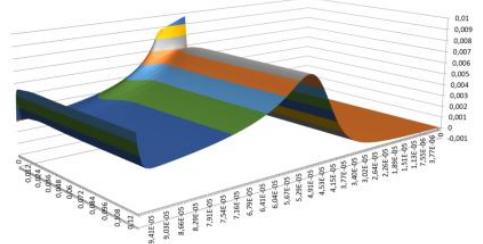

d)

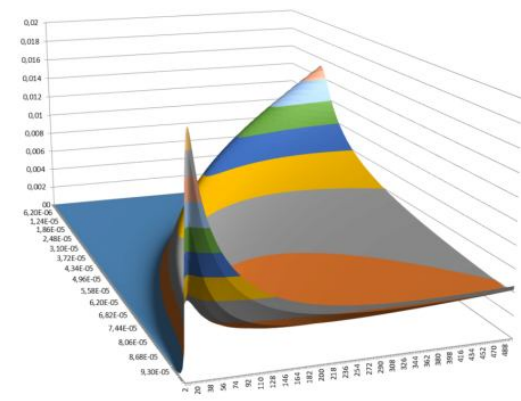

g)

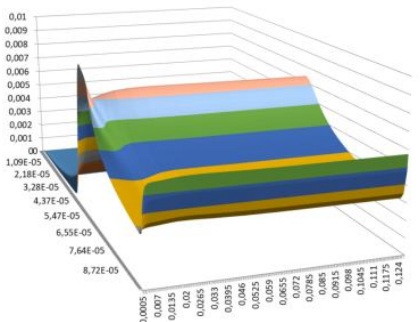

e)

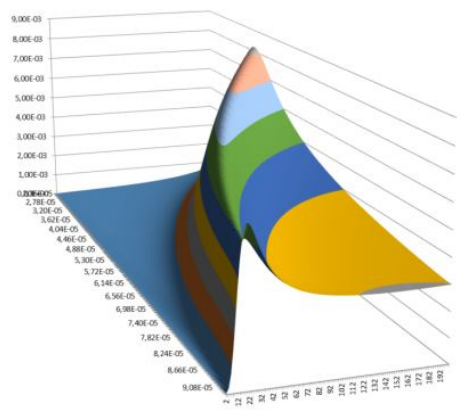

h)

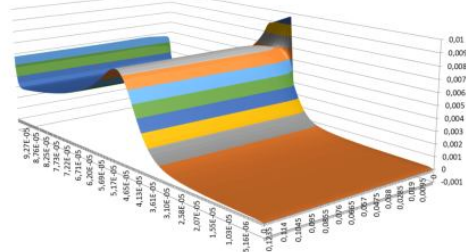

f)

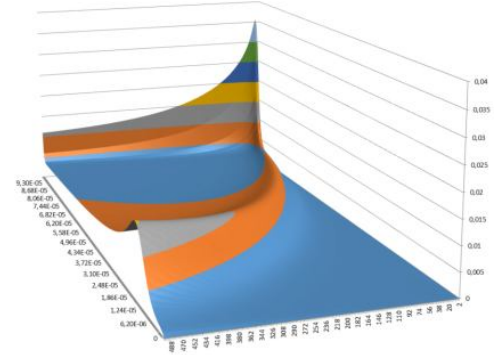

i)

Figure 2: plots at different angles: a), b), c) - for model with escalation, d), e), f) - for model with overlimiting initial conditions, g), h), i) for CVC model

$$
\frac{1}{F} \rho(t, x)=C_{1}(t, x)-C_{2}(t, x)
$$

Considerations, similar to the above mentioned, based on Figs. 2 d)-i) make it possible to substantiate Fig. 1 b)-d). It can be made in more details but less illustratively using the cross section of normalized current density.

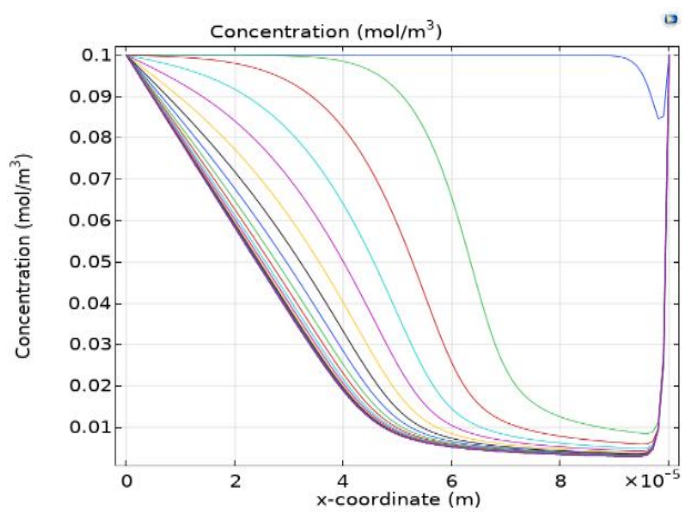

a)

\subsubsection{Major regularities of transfer}

Let us consider at first the distribution of cation and anion concentrations in various regions of diffusion layer.

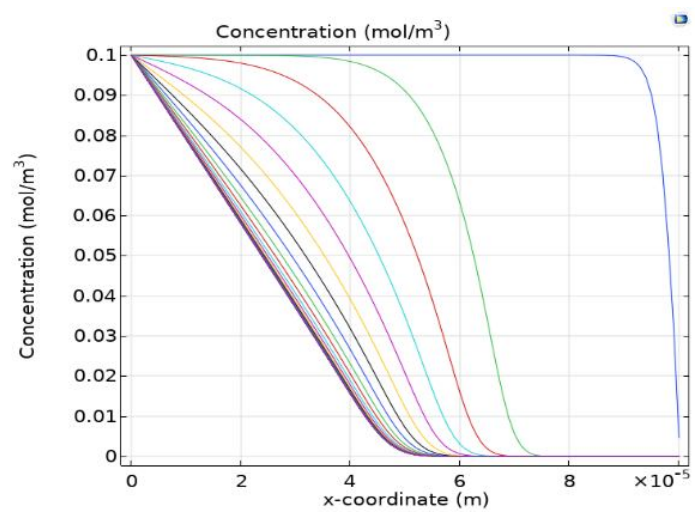

b) 


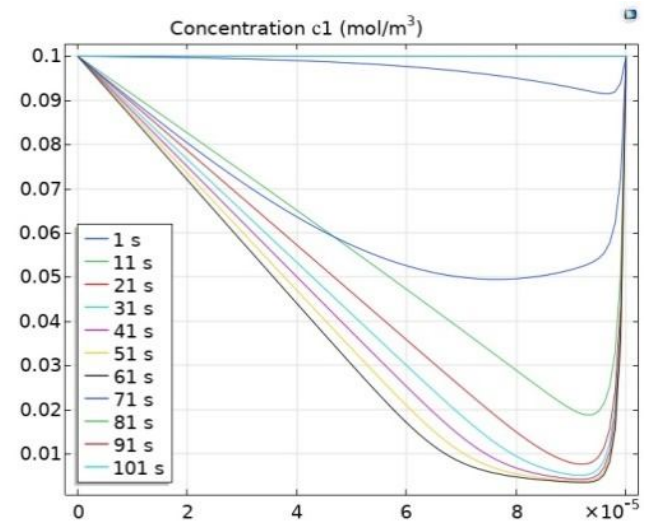

c)

Figure 3: Variation of cation and anion concentrations with regard to $x$

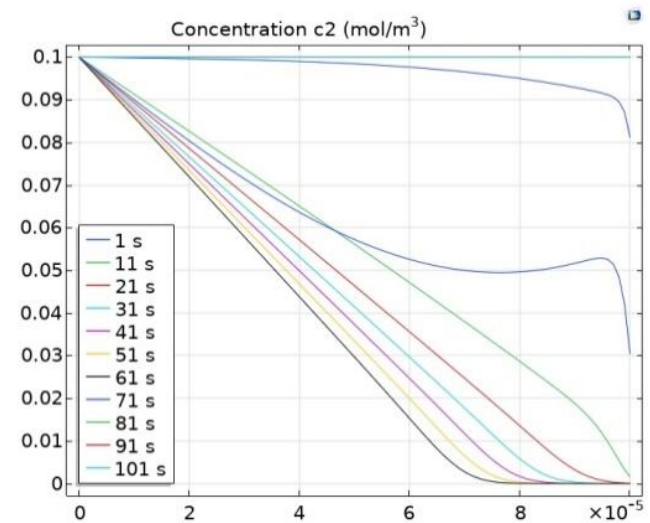

d) d) - corresponds to the model with zeroed potential jump

It can be seen in Fig. 3 that in quasi-equilibrium SCR, the anion concentration is zero with high accuracy, and the cation concentration rapidly reaches stationary regime. The maximum thickness of quasi-equilibrium SCR corresponds to the Debye length for this case. Distribution of concentration outside SCR in time becomes linear, and the cation concentration equals to the anion concentration with high accuracy $\left(C_{1}(t, x) \approx C_{2}(t, x)\right.$ ). In the extended SCR, the cation and anion concentrations rapidly vary, herewith, $C_{1}(t, x)>>C_{2}(t, x)$. Thus, it is possible to conclude that in the quasi-equilibrium SCR near membrane, the cation

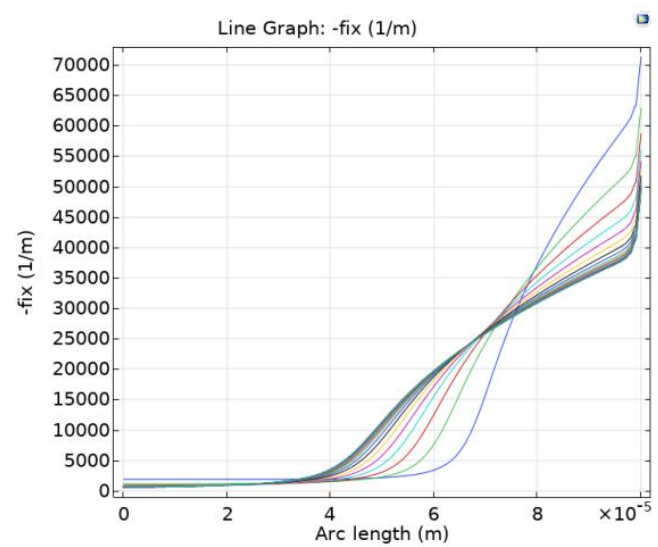

a)

Figure 4: Cross-section in electric field intensity in the ENR with regard to $x$ at fixed times: a) and b) - for model with escalation

The plot of electric field intensity (Fig. 4 a) in the quasi-equilibrium region varies very quickly, remaining convex function, and in the extended region of space charge, the plot is concave, being gradually stabilized. The intensities concentration varies rapidly with regard to $x$, and the anion concentration is zero (if the current is higher than the ultimate value), and in the extended SCR, the cation concentration rapidly decreases, still remaining higher than the anion concentration. In the ENR, the cation and anion concentrations vary sufficiently slowly, gradually approaching stationary linear distribution.

Let us consider the plots of electric field intensity. Conclusions, similar to the above mentioned, are possible with regard to variation of cross section of electric field intensity (Fig. 4).

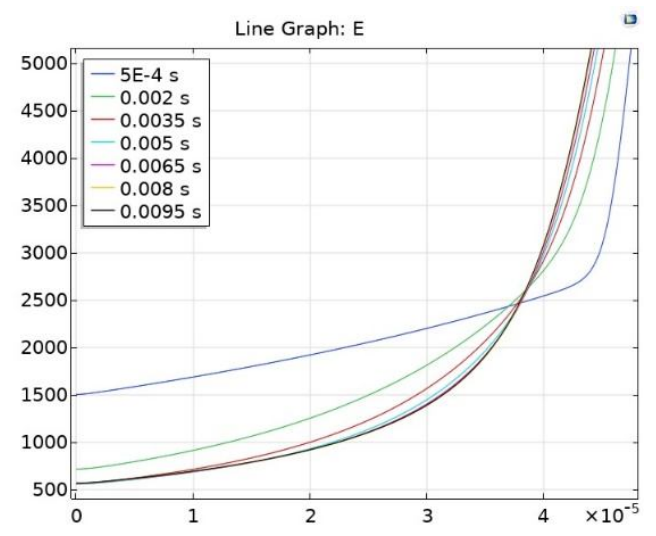

b)

slowly increase in the ENR from $10^{2} \mathrm{~V} / \mathrm{m}^{2}$ and sharply in the SCR, reaching $10^{5} \mathrm{~V} / \mathrm{m}^{2}$. 


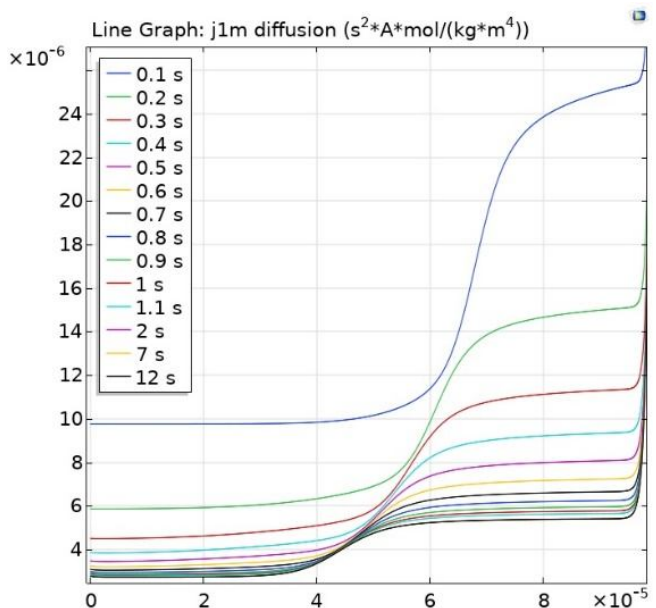

a)

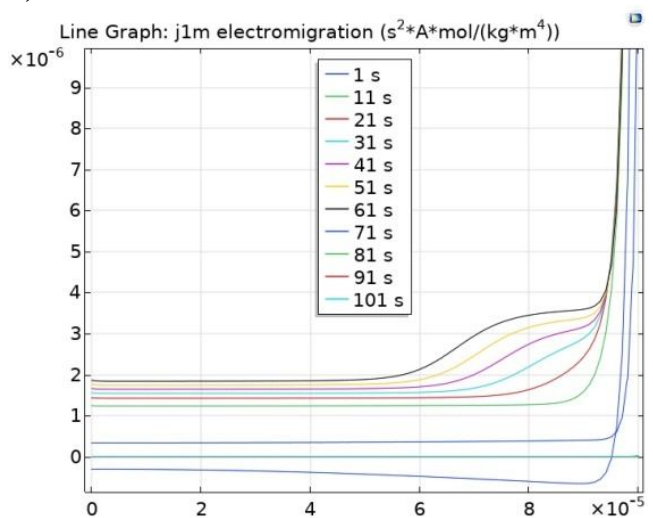

c)

Figure 5: Cross section of cation flow with regard to $x$ in fixed times corresponding to the model with zeroed potential jump: a) electromigration portion of flow, b) diffusion part of flow

In all cases, except for the problem of discharge of space charge and the problem with periodic variation of potential jump, the flow $j_{1}$ is the increasing function, which is convex in the ENR and concave in the extended region of space charge, there are oscillations in quasi-equilibrium region caused by discordance between the initial condition for concertation and the boundary condition at $x=H$. With the time increase, the plot of flow $j_{1}$ gradually becomes constant (Fig. 5 a) and b)). The flow $j_{2}$ is significantly lower than $j_{l}$, and with increase in time, it approaches zero. It should be mentioned that in all cases in extended SCR, the electric migration flow is by an order of magnitude higher than diffusion (Fig. 5 a) and b)). Therefore, the extended region can be referred to as the region of electric migration.

In the problem of discharge of space charge, after zeroing of potential jump, the values of cation and anion flows are approximately equal, moreover, the diffusion and the electric migration flows are approximately equal (Fig.5 c) and d)). Due to these flows the space charge disappears very quickly (in seconds).

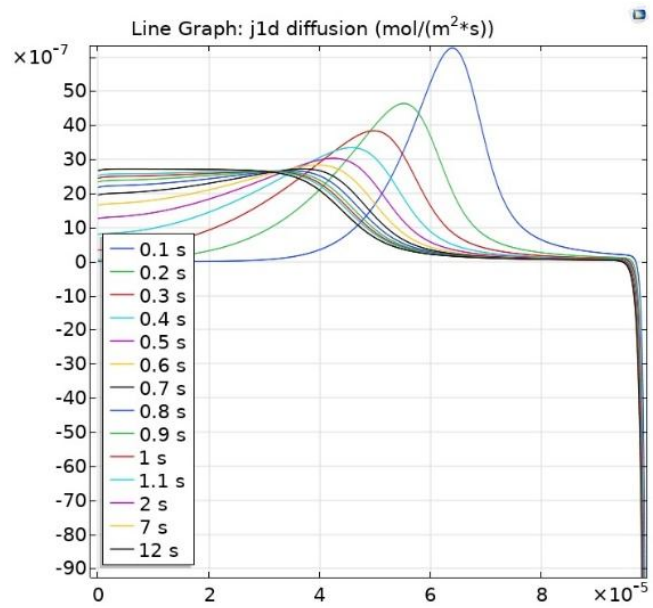

b)

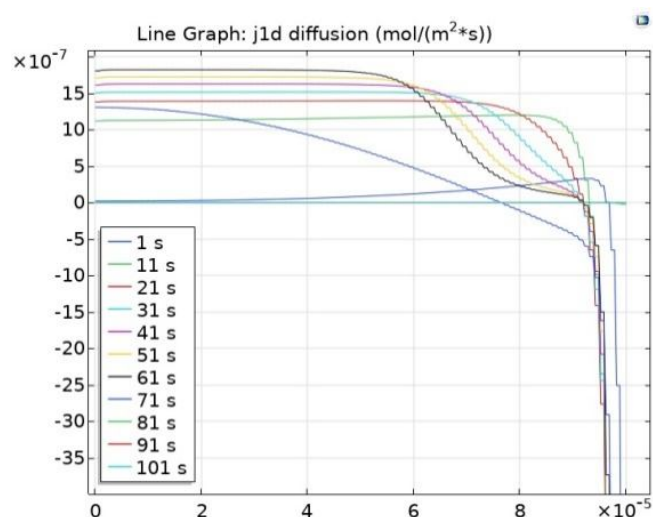

d)

\subsubsection{Numerical analysis of stability of overlimiting regime}

Let us analyze numerically the stability of overlimiting regime with regard to oscillations of potential jump. With this aim, let us preset the oscillations of potential jump in the form of $\varphi(t, H)=\varphi_{0}+d \cdot \sin (\omega t)$, where $\varphi_{0}, d, \omega$ are the jump potential corresponding to overlimiting regime, the amplitude and the frequency of oscillations, respectively, and calculate the respective oscillations of current density. Table 1 summarizes the calculation results at $\phi_{0}=-1.5$ and $\omega=1$, and various $d$.

Table 1: Current density as a function of oscillations of potential jump

\begin{tabular}{|l|l|l|l|l|}
\hline \multicolumn{1}{|c|}{$\mathrm{d}$} & 0.05 & $\begin{array}{l}0.0 \\
1\end{array}$ & $\begin{array}{l}0.00 \\
5\end{array}$ & 0.001 \\
\hline Oscillation amplitude, \% & 4.8 & 1 & 0.5 & 0.1 \\
\hline Iav, \% & 6.5 & 1.3 & 0.7 & 0.1 \\
\hline
\end{tabular}

It can be seen in Table 1 that decrease in oscillations of potential jump leads to decrease in oscillations of current density. Hence, it is possible to conclude that the 
nonstationary overlimiting transfer is stable with regard to oscillations of potential jump.

\subsubsection{Peculiar Features of Nonstationary Superlimiting overlimiting Regime and Algorithm of Approximate Analytical Solution}

The performed numeric analysis has made it possible to reveal peculiarities of nonstationary overlimiting transfer and to propose algorithm of approximate analytical solution to this problem:

1) Diffusion layer is subdivided into the following regions: ENR, extended SCR, quasi-equilibrium SCR, and intermediate layer between ENR and extended SCR, in each of these regions, equations are simplified in their own ways. Similar subdivision was used $\mathrm{in}^{3}$ for approximate asymptotic solution to stationary boundary problem. In this case, the boundaries of regions vary in time;

2) In the ENR, the problem is significantly simplified, its solution is already known; ${ }^{1,8}$

3) As shown above, in quasi-equilibrium SCR, the solution actually does not depend on time and flows, hence, it is possible to use the Debye-Hückel solution to the problem of stationary double electric layer;

4) In extended SCR, the flows actually do not depend on $x$ and concentration on $t$, electric migration prevails over diffusion transfer, therefore, in the first approximation, the following set of equations can be applied:

5)

$$
\begin{gathered}
j_{i}=j_{i}(t) \quad i=1,2 \\
j_{i}=-\frac{F}{R T_{0}} z_{i} D_{i} C_{i} E \quad i=1,2 \\
\frac{\partial E}{\partial x}=\frac{F}{\varepsilon_{a}}\left(C_{1}-C_{2}\right) \\
E=\sqrt{-\frac{2 R T_{0}}{\varepsilon_{a}}\left(\frac{j_{1}}{D_{1}}+\frac{j_{2}}{D_{2}}\right) x+\theta(t)} \\
C_{i}=-\frac{j_{i}}{z_{i} D_{i}} \frac{R T_{0}}{F} \frac{1}{E} \quad i=1,2
\end{gathered}
$$

6) The intermediate region confined by dashed line in Fig. 1 is small, it can be neglected in the first approximation;

Therefore, the main problem is in analytical determination of region boundaries by combination of solutions from adjacent regions, or by numerical solution.

\section{CONCLUSION}

This work studied nonstationary transfer of 1:1 electrolyte in donating layer near CEM in potentiostatic overlimiting regime. The main regularities of ion transfer of 1:1 salt have been determined. It has been demonstrated that the diffusion layer is comprised of the ENR and the SCR, which in its turn is comprised of extended region, the sizes of which are lower than those of diffusion layer but comparable with it, and moderate boundary layer near CEM. The sizes of extended SCR depend on the ratio of current density in circuit to ultimate current, whereas the size and salt ion transfer in boundary layer near CEM do not depend on current density and are entirely determined by exchange capacity of CEM and its selective properties. The main regularities of nonstationary transfer of binary electrolyte in depleted diffusion layer have been determined, namely: its structure, distribution of concentrations, electric field intensity, etc. The nonstationary problems of transfer in diffusion layer are classified in terms of various criteria.

\subsection{Classification in Terms of Potential Jump:}

1. Potentiostatic regime, constant potential jump:

a) sublimiting regime

b) overlimiting regime

2. Potentiodynamic regime, alternating potential jump

a) sublimiting regime

b) overlimiting regime

c) mixed regime, when some potential jumps are referred to sublimiting regime, and some - to overlimiting regime. This can be exemplified by calculation of $\mathrm{CVC}$, when potential jump varies linearly from zero to infinity. Another example is analysis of pulse regime, when current in piecewise manner equals, for instance, to zero and is higher or lower than the ultimate current. The third example is reverse electrodialysis, when current becomes opposite, etc.

\subsection{Classification in Terms of Initial Conditions for Concentrations and Potential:}

1. Constant values, such formulation of conditions is reasonable, for instance, upon analysis of initial segment of desalination channel of electrodialysis apparatus. This can be exemplified by determination time of establishment of stationary regime, analysis of generation and development of space charge, etc.

2. Variable initial conditions, that is, the initial conditions depend on $x$. This can be exemplified by simulation of transfer far from the start of channel, when the profiles of concentrations and potentials are already developed and can correspond to both sublimiting regime and to overlimiting regime.

\subsection{Peculiar Problems}

1. The interest is attracted to the regime when under sublimiting initial conditions, overlimiting potential jump is preset for concentration and potential, or vice versa. 
2. Periodic regime when the potential jump varies periodically, and the initial conditions are coordinated with it. This can be exemplified by calculation of impedance.

\section{ACKNOWLEDGMENTS}

This work was supported by the Russian Foundation for Basic Research, project No. 20-58-12018 NNIO_a: The influence of electroconvection, water dissociation, and geometry of spacers on electrodialysis desalination in intensive current regimes.

\section{REFERENCES}

1. V.I. Zabolotskii, V.V. Nikonenko, Perenos ionov $\mathbf{v}$ membranakh [Ion transfer in membranes]. Moscow: Nauka, 1996.

2. I. Rubinstein, L. Shtilman, Voltage against current curves of cation exchange membranes, Journal of the Chemical Society, Faraday Transactions, no. 75, pp. 231-246, 1979.

3. N.O. Chubyr', A.V. Kovalenko, M.Kh. Urtenov, Chislennye i asimptoticheskie metody analiza perenosa 1:1 elektrolita $v$ membrannykh sistemakh [Numerical and asymptotic methods of analysis of transfer of 1:1 electrolyte in membranes]. Krasnodar, 2018.

4. V.A. Babeshko, V.I. Zabolotskii, M.A.Kh. Urtenov, R.R. Seidov, N.M. Seidova. Dekompozitsionnye uravneniya dlya statsionarnogo perenosa elektrolita $v$ odnomernom sluchae [Decomposition equations for stationary transfer of electrolyte in 1D case], Elektrokhimiya, vol. 33, no. 8, pp. 855-862, 1997.

5. A. Uzdenova, A. Kovalenko, M. Urtenov, V. Nikonenko, 1D mathematical modelling of nonstationary ion transfer in the diffusion layer adjacent to an ion-exchange membrane in galvanostatic mode, Membranes, vol. 8, no. 3, p. 84, 2018.

6. J.A. Manzanares, W.D. Murphy, S. Mafe, H.Reiss, Numerical Simulation of the Nonequilibrium Diffuse Double Layer in Ion-Exchange Membranes, The Journal of Physical Chemistry, no. 97, pp. 8524-8530, 1993.

https://doi.org/10.1021/j100134a023

7. R. Femmer, A. Mani, M. Wessling, Ion transport through electrolyte/polyelectrolyte multi-layers, Scientific Reports, 5, 11583 (2015).

8. J. Newman, K.E. Thomas-Alyea, Electrochemical Systems, Honoken, NJ: Wiley \& Sons, Inc, 2004.

9. S.M. Davidson, M. Wessling, A. Mani, On the Dynamical Regimes of Pattern-Accelerated Electroconvection. Sci. Rep., no. 6, 2016.

https://doi.org/10.1038/srep22505 